\title{
Confinement of electrons in size-modulated silicon nanowires
}

\author{
S. Cahangirov ${ }^{1}$ and S. Ciraci ${ }^{1,2, *}$ \\ ${ }^{1}$ UNAM-Institute of Materials Science and Nanotechnology, Bilkent University, Ankara 06800, Turkey \\ ${ }^{2}$ Department of Physics, Bilkent University, Ankara 06800, Turkey \\ (Received 3 March 2009; revised manuscript received 3 June 2009; published 12 August 2009)
}

\begin{abstract}
Based on first-principles calculations we showed that superlattices of periodically repeated junctions of hydrogen-saturated silicon nanowire segments having different lengths and diameters form multiple quantumwell structures. The band gap of the superlattice is modulated in real space as its diameter does and results in a band gap in momentum space which is different from constituent nanowires. Specific electronic states can be confined in either narrow or wide regions of superlattice. The type of the band lineup and hence the offsets of valence and conduction bands depend on the orientation of the superlattice as well as on the diameters of the constituent segments. Effects of the $\mathrm{SiH}$ vacancy and substitutional impurities on the electronic and magnetic properties have been investigated by carrying out spin-polarized calculations. Substitutional impurities with localized states near band edges can make modulation doping possible. Stability of the superlattice structure was examined by ab initio molecular-dynamics calculations at high temperatures.
\end{abstract}

DOI: 10.1103/PhysRevB.80.075305

PACS number(s): 73.63.Nm, 73.22.-f, 75.75.+a

\section{INTRODUCTION}

Rodlike $\mathrm{Si}$ nanowires (SiNW) have been synthesized down to $\sim 1 \mathrm{~nm}$ diameter. ${ }^{1}$ They are attractive onedimensional materials because of the well-known silicon fabrication technology that make them directly usable on the Si-based chips. Even if unsaturated dangling bonds on the outer surface usually attribute a metallic character to SiNWs, they become insulator (or semiconductor) upon saturation of these dangling bonds by hydrogen atoms. ${ }^{2}$ SiNWs display diversity of electronic properties depending on their diameter as well as their orientation. In particular, the band gap of semiconductor SiNWs varies with their diameters. They can be used in various electronic, spintronic, and optical applications, such as field-effect transistors, ${ }^{3}$ light-emitting diodes, ${ }^{4}$ lasers ${ }^{5}$ and interconnects. The conductance of these semiconductor nanowires can be tuned easily by doping ${ }^{6,7}$ during the fabrication process or by applying a gate voltage. Recent studies have shown that $3 d$ transition-metal-doped Si nanowires become half metallic. ${ }^{8}$

This paper demonstrates that SiNWs of different diameters can form stable superlattices. The electronic band structure of the superlattice is different from the constituent SiNWs and is modulated in real space leading to a multiple quantum structure and/or to a series of quantum dots. In these size-induced quantum wells, specific states are confined. One-dimensional multiple quantum-well structures generated by compositional modulation of nanowires were examined previously. For example, superlattices of $\mathrm{GaAs} / \mathrm{GaP},{ }^{9}$ InAs/InP, ${ }^{10}$ and $\mathrm{Si} / \mathrm{SiGe}$ (Ref. 11) nanowires were fabricated. Moreover, superlattices of $\mathrm{Si}$ and $\mathrm{Ge}$ were investigated theoretically. ${ }^{12}$ In these structures multiple quantum wells are formed because of the different nature of materials that constitute the nanowire superlattice. In the present work however, multiple quantum wells are formed because of the quantum-size effect, which is a diversification of the electronic structure of the same material with a change in its size.

Our results are obtained by performing first-principles plane-wave calculations within density-functional theory
(DFT) (Ref. 13) using ultrasoft pseudopotentials. ${ }^{14,15}$ The exchange-correlation potential has been approximated by generalized gradient approximation using PW91 functional. ${ }^{16} \mathrm{~A}$ plane-wave basis set with kinetic-energy cutoff of up to $250 \mathrm{eV}$ has been used. All atomic positions and lattice constants are optimized. The convergence for energy is chosen as $10^{-5} \mathrm{eV}$ between two steps and the maximum force allowed on each atom is $0.02 \mathrm{eV} / \AA$. More details about calculations can be found in Ref 8 .

\section{SIZE-MODULATED SILICON NANOWIRES}

Here Si nanowires (i.e., $\mathrm{Si}_{N_{1}}$ with diameter $\sim D_{1}$ and having $N_{1} \mathrm{Si}$ atoms in the primitive unit cell) are first cut from the ideal bulk crystal along desired direction. Then in every alternating segment comprising $l_{1}$ unit cell the diameter $D_{1}$ is kept fixed but in the adjacent segment comprising $l_{2}$ unit cell (each having $N_{2} \mathrm{Si}$ atoms) the diameter is reduced to $D_{2}$. The latter part can be identified as the segment of $\mathrm{Si}_{N_{2}}$ nanowire. At the end, the segments of $\mathrm{Si}_{N_{1}}$ and $\mathrm{Si}_{N_{2}}$ have made a smooth junction and hence formed an ideal superlattice $\mathrm{Si}_{N}$ so that its diameter is modulated in real space. Note that $N$ $\leq l_{1} N_{1}+l_{2} N_{2}$ because some surface atoms attaching with a single bond to the surface were removed at the beginning. Subsequently we relaxed the atomic structure of this bare $\mathrm{Si}_{N}$. Upon relaxation, the dangling bonds on the surface are saturated by $\mathrm{H}$ atoms and $\mathrm{Si}_{N} \mathrm{H}_{M}$ superlattice is further relaxed for final atomic structure and the lattice constant. The resulting superlattice can be described by a Si rod with alternating diameters or a nanowire with alternating wide and narrow parts. Here we consider two superlattice structures, namely, $\mathrm{Si}_{138} \mathrm{H}_{42}$ and $\mathrm{Si}_{157} \mathrm{H}_{64}$, which are grown in [111] and [100] directions, respectively. For the latter we investigate the surface defect and also boron, B and phosphorus, $\mathrm{P}$ substitutional impurities.

\section{A. $\mathrm{Si}_{138} \mathrm{H}_{42}$ superlattice}

To form the superlattice, $\mathrm{Si}_{138} \mathrm{H}_{42}$, we took $l_{1}=1$ unit cell of $\mathrm{Si}_{68}$ and $l_{2}=2$ unit cell of $\mathrm{Si}_{38}$ cut in [111] direction of the 


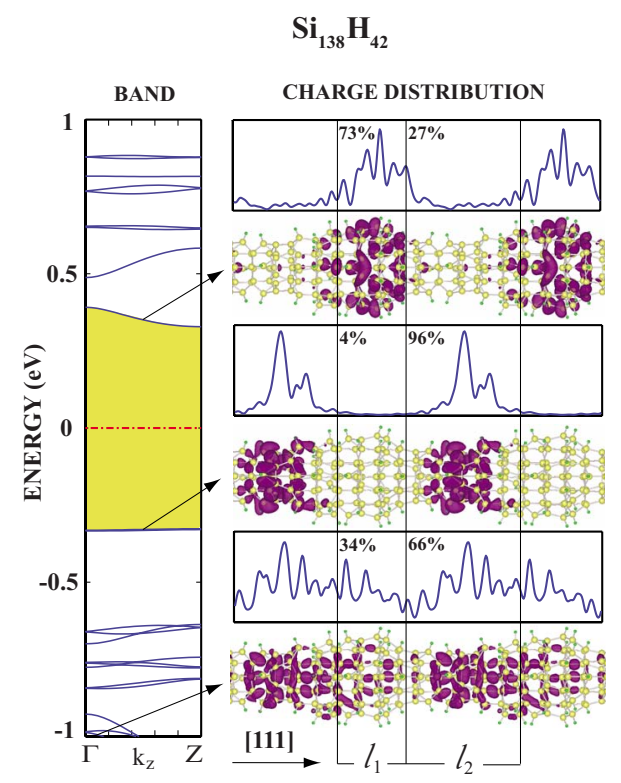

FIG. 1. (Color online) Energy-band structure of the superlattice, $\mathrm{Si}_{138} \mathrm{H}_{42}$, formed from periodically repeated junctions consisting of one unit cell of SiNW with $D_{1} \sim 11 \AA$ and two unit cell of SiNW with $D_{2} \sim 7 \AA$ formed along [111] direction. Here the diameter $D$ is defined as the largest distance between two $\mathrm{Si}$ atoms in the same cross-sectional plane. Isosurface charge densities of specific bands and their planarly averaged distribution along the superlattice axis together with their confinement in percentages are also shown. Large and small balls indicate $\mathrm{Si}$ and $\mathrm{H}$ atoms. Zero of energy is set at the Fermi level shown by dash-dotted line.

bulk silicon crystal. The combined structure had six $\mathrm{Si}$ atoms attached to the surface by only one bond. These atoms were removed at the beginning and the structure was optimized. After the relaxation, all $\mathrm{Si}$ atoms were at least triply coordinated. The dangling bonds of triply coordinated $\mathrm{Si}$ atoms were saturated with hydrogen atoms and the structure was relaxed again. The band structure and isosurface charge densities of the resulting superlattice is shown in Fig. 1. Propagating states with dispersive bands have charge densities distributed everywhere in the superlattice rod. For example, a state near $\sim-1 \mathrm{eV}$ is a propagating state. The states of flat degenerate band at the top of the valence band are confined in the narrow segment. The integral of the planarly averaged charge, $\int_{l_{1}}|\overline{\Psi(z)}|^{2} d z=0.96$ indicates a rather strong confinement. The states of the lowest conduction band with low dispersion are localized in the wide segment with relatively weaker confinement. This situation implies the staggered band alignment with confined hole states in the narrow part and confined electrons in the wide part. Confinement may increase with the size (length) of the confining segment since the energy of the state relative to the barrier varies. For perfect localization, i.e., $\sim 100 \%$ confinement in $l_{1}$ or $l_{2}$, where coupling between confined states are hindered, the segment $l_{1}$ or $l_{2}$ behaves as a quantum dot. Confinement and bandedge alignment in the present superlattice are reminiscent of the two-dimensional pseudomorphic or commensurate semiconductor superlattices. ${ }^{17}$

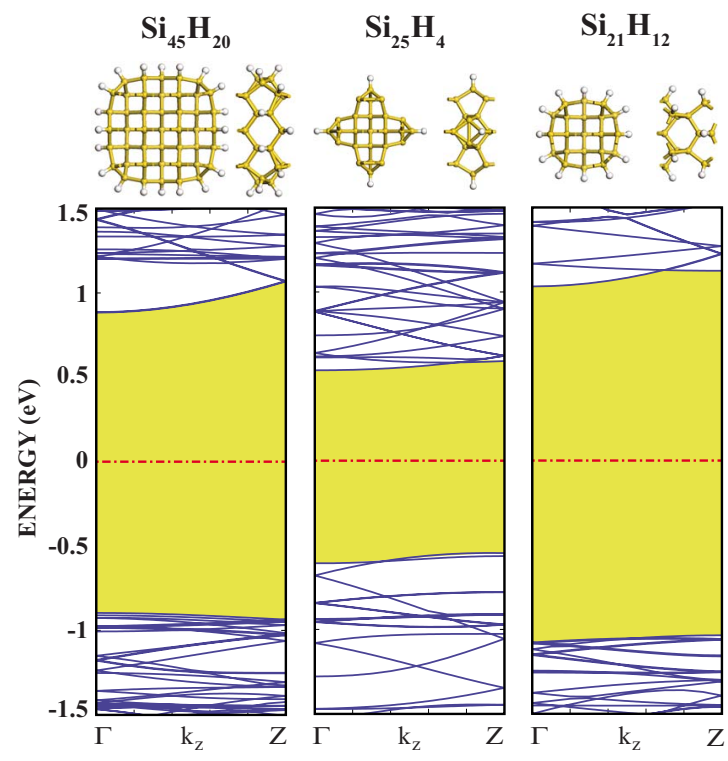

FIG. 2. (Color online) Band structures of infinite and periodic $\mathrm{Si}_{45} \mathrm{H}_{20}, \mathrm{Si}_{25} \mathrm{H}_{4}$, and $\mathrm{Si}_{21} \mathrm{H}_{12}$ nanowires, which constitute $\mathrm{Si}_{157} \mathrm{H}_{64}$ structure. The front and side view of these relevant structures are shown on the top of the band-structure plots. Numerals as subscripts of $\mathrm{Si}$ and $\mathrm{H}$ indicate the number of $\mathrm{Si}$ and $\mathrm{H}$ atoms in the unit cell. Energy-band gaps are shaded.

\section{B. Pristine $\mathrm{Si}_{157} \mathrm{H}_{64}$ superlattice and vacancy}

Here we presents the superlattice formation and confined states in a different structure, namely, $\mathrm{Si}_{157} \mathrm{H}_{64}$. To form this structure we took $l_{1}=2$ unit cell of $\mathrm{Si}_{45}$ and $l_{2}=3$ unit cell of $\mathrm{Si}_{25}$ cut in [100] direction of the bulk silicon crystal. The combined structure had eight silicon atoms that were attached to the surface by only one bond. We have removed these atoms at the beginning and relaxed the resulting structure. Then we have passivated the dangling bonds of the final structure and relaxed the whole structure again. As a result, the wide part of the nanowire superlattice had $l_{1}=2$ unit cell of $\mathrm{Si}_{45} \mathrm{H}_{20}$ with $D_{1} \sim 14 \AA$ and the narrow part had $l_{2}$ $=3$ unit cell of $\mathrm{Si}_{25} \mathrm{H}_{4}$ and $\mathrm{Si}_{21} \mathrm{H}_{12}$ mixture with $D_{2} \sim 7 \AA$. Figure 2 presents the band structure of the corresponding infinite periodic nanowires which constitute the $\mathrm{Si}_{157} \mathrm{H}_{64}$ superlattice structure. To compare with the band structure of the superlattice (presented in Fig. 3 as pristine), the band structure of these nanowires were folded $l_{1}+l_{2}=5$ times. One can see that the band gap of the superlattice is close to the lowest band gap of its constituents. This is expected for the superlattice structures having normal band lineup. The band gaps of structures shown in Fig. 2 do not obey the wellknown trend, $E_{G}=C / D^{\alpha}+E_{G}[$ bulk $\mathrm{Si}]$, occurring due to the quantum-confinement effect ${ }^{18}$ at small $D$. We ascribe this result to the surface reconstructions which differ in accordance with the procedure of atomic relaxation. Quantumconfinement trend occurs when Si nanowires, cut from ideal bulk, are directly passivated with $\mathrm{H}$ atoms and subsequently relaxed. In the present study, however, the ideal structures are first relaxed, then passivated with $\mathrm{H}$ atoms, and then relaxed again to obtain the final structure. ${ }^{8}$ Such Si nanowire structures with $D \sim 1 \mathrm{~nm}$ may not obey the quantum- 


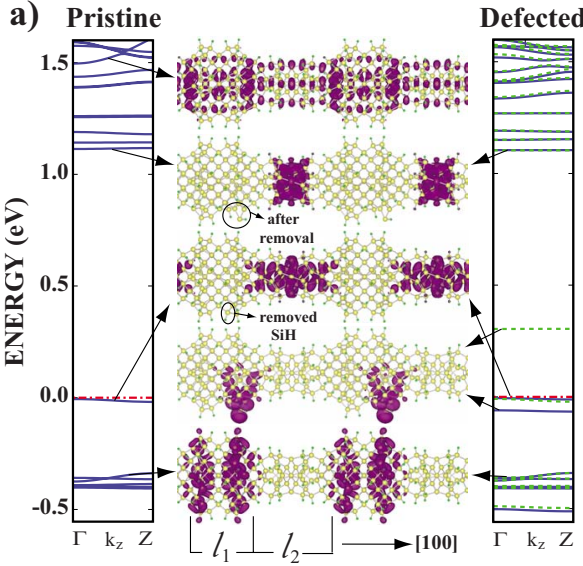

b) INTERATOMIC DISTANCE DISTRIBUTION

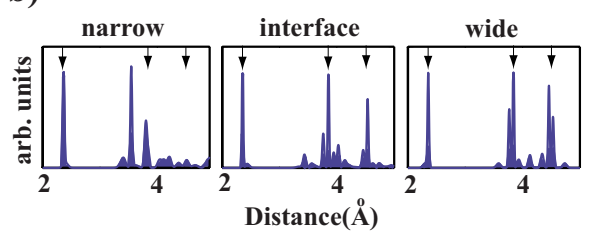

FIG. 3. (Color online) (a) Electronic structure and isosurface charge densities of selected states for pristine $\mathrm{Si}_{157} \mathrm{H}_{64}$ and defected $\mathrm{Si}_{156} \mathrm{H}_{63}$ (with one $\mathrm{Si}-\mathrm{H}$ on the surface removed) nanowire superlattices. Arrows pointing at the same isosurface charge-density plot indicates that those states are not affected by the formation of the defect and have nearly the same charge-density profile. Fermi level, represented by dashed-dotted (red) line, was shifted to the valenceband edge and set to zero. Solid (blue) and dashed (green) lines in the right-hand side box represent the majority- and minority-spin bands, respectively. (b) The distribution of interatomic distances of relaxed superlattice. The arrows indicate the ideal bulk first-, second-, and third-nearest-neighbor distances.

confinement trend. The decline of the gap values from the $1 / D^{\alpha}$ behavior has been also reported for other types of SiNWs and was attributed to surface effects. ${ }^{19}$

Figure 3(a) presents the band structure and the projected charge-density isosurface plots of pristine $\mathrm{Si}_{157} \mathrm{H}_{64}$ and defected $\mathrm{Si}_{156} \mathrm{H}_{63}$ nanowire superlattices. Flat minibands of $\mathrm{Si}_{157} \mathrm{H}_{64}$ structure near the edge of conduction and valence bands are distinguished. ${ }^{17}$ In contrast to propagating states of dispersive bands near $1.5 \mathrm{eV}$, the states of these flat minibands are confined in the narrow segments of the superlattice. This situation also implies a normal band lineup. We are aware of the fact that fabrication of nanowire superlattices having ideally tapered as in our case is impossible. So we examined the effect of an imperfection on the behavior of the system by removal of a $\mathrm{Si}-\mathrm{H}$ atom pair, which was normally attached to the surface. The location of this defect and structural change after relaxation is given in Fig. 3(a). The resulting structure has odd number of electrons, so we made spinpolarized calculation. One can see that the valence- and conduction-band edges are not affected by defect formation while one empty and one filled state localized around the defected area appear near the valence-band edge. The magnetic moment induced by the defect formation is $1 \mu_{B}$. The vacancy states in bulk $\mathrm{Si}$ are located deep in the band gap. In the superlattice structure, this vacancy state is located near the valence band since the defect is formed on the surface at the interface between two segments having different diameters.

In Fig. 3(b) the distribution of interatomic distances shows significant reconstruction and deformation. In particular, owing to increased surface/volume ratio the deviation of the second- and third-nearest-neighbor distances from the bulk crystal is rather large at the narrow and interface regions. We note that in both short periodicity superlattices discussed above the electronic structures are significantly different from the constituent SiNWs. As $l_{1}$ and/or $l_{2}$ increases, the superlattice effects and confined states may become more pronounced, and band offset converges to a welldefined value. When the lengths of $l_{1}$ and $l_{2}>\lambda_{B}$ (Broglie wavelength), the electronic properties in each segment becomes close to those in constituent nanowires. In addition to $l_{1}$ and $l_{2}, D_{1}$ and $D_{2}$ and also the direction of growth are also critical parameters, since the electronic structure of the constituent SiNWs is strongly dependent on these parameters. As $D_{1}$ and $D_{2}$ increases, the surface effects decrease and the density of states becomes more bulklike. The discontinuities of valence and conduction bands of the superlattice is expected to diminish since the band gap of the constituent nanowires converge to the bulk $\mathrm{Si}$ value. However for a significant $\Delta D=D_{1}-D_{2}$ the superlattice effects, in particular, confinement of specific states are expected to continue. In contrast, smooth interfaces leading to hornlike profiles can be used for focused electron emission. ${ }^{20}$ Recently, transport properties of size-modulated SiNWs have been shown to depend on the growth direction. ${ }^{21}$

The stability of the $\mathrm{Si}_{157} \mathrm{H}_{64}$ structure was analyzed using the finite-temperature $a b$ initio molecular-dynamics calculations with time steps of $2 \times 10^{-15}$ s. Figure 4(a) presents the total-energy difference of the system with respect to the ground-state energy at $0 \mathrm{~K}$ as a function of time. In the first calculation we have raised the temperature of the system from 500 to $1400 \mathrm{~K}$ in 2 ps. During this calculation there was no major change in the structure and the interface region was not destroyed by diffusion. In the second calculation we took atomic positions and velocities of the system from the first calculation when the system was at $1300 \mathrm{~K}$ and kept the temperature constant for 9 ps. This calculation also resulted in no major change in the structure of the superlattice. Figures 4(b) and 4(c) present the path of Si atoms during the second calculation at the surfaces of wide and narrow parts, respectively. The range of the thermal fluctuations are nearly the same and span a diameter of about $1 \AA$. Figures $4(\mathrm{~d})$ and 4(e) present the path of Si atoms at the center of the wide and narrow parts, respectively. As seen the range of thermal fluctuations in the narrow part $(\sim 0.9 \AA)$ is larger than that in the wide part $(\sim 0.5 \AA)$. It should be noted that, the temporal trajectories of $9 \mathrm{ps}$ in the present $a b$ initio moleculardynamics calculations are rather long as compared to ones usually carried out in the stability tests but may not be long enough to accumulate necessary statistics. However, the temporal trajectories at temperature as high as $1300 \mathrm{~K}$ in the present study are long enough to eliminate the existence of any atomic configuration in a weak local minimum of the Born-Oppenheimer surface, which is prone to structural instability. Therefore, based on the accurate structure optimi- 

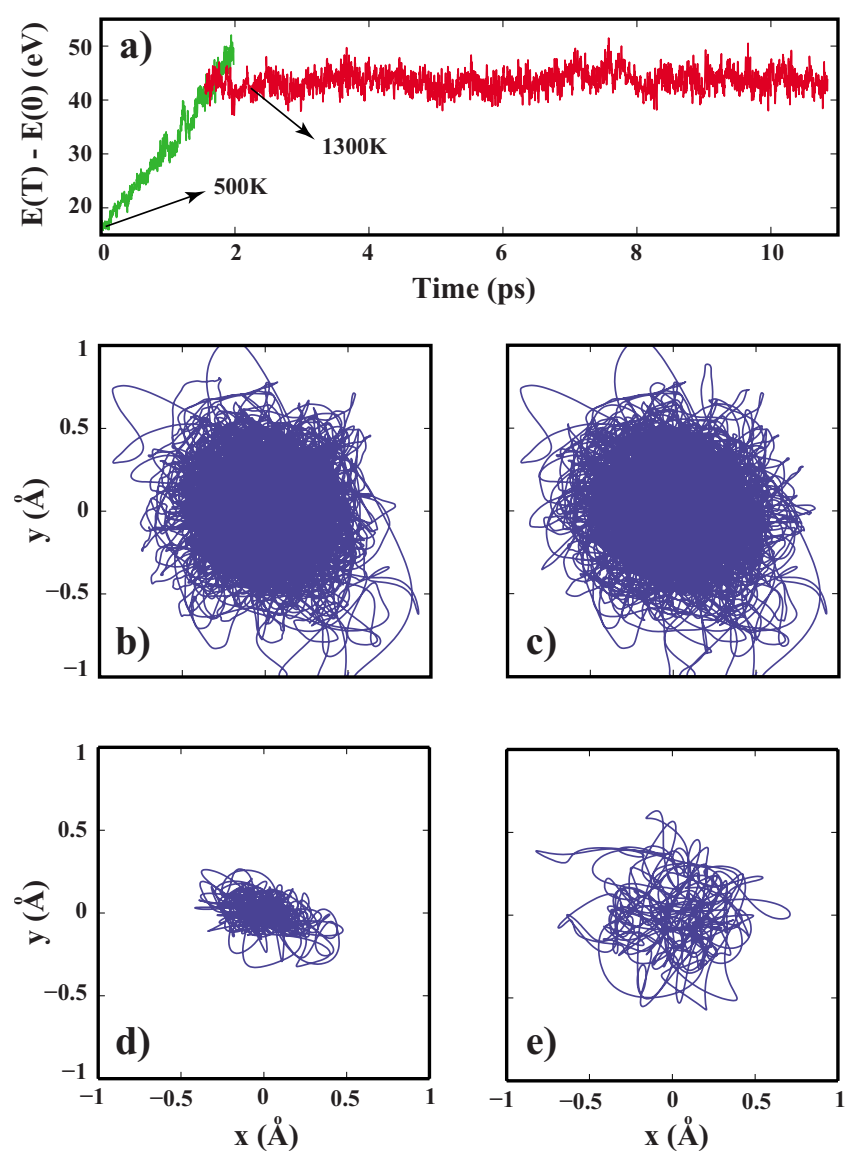

FIG. 4. (Color online) (a) Energy per unit cell of $\mathrm{Si}_{157} \mathrm{H}_{64}$ superlattice as its temperature is increased from 500 to $1400 \mathrm{~K}$ (green/ light) and kept constant at $1300 \mathrm{~K}$ (red/dark). Trajectories of atoms at the surface of (b) wide and (c) narrow parts and at the center of (d) wide and (e) narrow parts of the superlattice, projected on a plane perpendicular to the wire axis, as the system is kept at 1300 K.

zation and temporal trajectories as long as 9 ps carried out at $1300 \mathrm{~K}$, we believe that superlattice structure under study is stable at least at a temperature higher than the usual operation temperature $(\sim 425 \mathrm{~K})$ of a device.

\section{C. $B$ and $P$ doping of $\mathrm{Si}_{157} \mathrm{H}_{64}$ superlattice}

Next we examine the effect of doping of the $\mathrm{Si}_{157} \mathrm{H}_{64}$ superlattice by substitutional B and $\mathrm{P}$ impurities. Both structures have odd number of electrons and hence spin-polarized calculations have been carried out. Nevertheless, B-doped structure resulted in a nonmagnetic state with half-filled band edge. P-doped structure resulted in a magnetic state with a magnetic moment of $1 \mu_{B}$. In Fig. 5 the electronic structure of pristine and doped superlattices are shown to follow the effect of the doping on the states at the band edges. Owing to the small diameter of the superlattice, the band structure is affected by the impurity. Since the band gap of the wide part is larger than that of the narrow part, the state associated with the impurity, which occurs normally either near the valence or conduction band, falls in the bands of the narrow part.
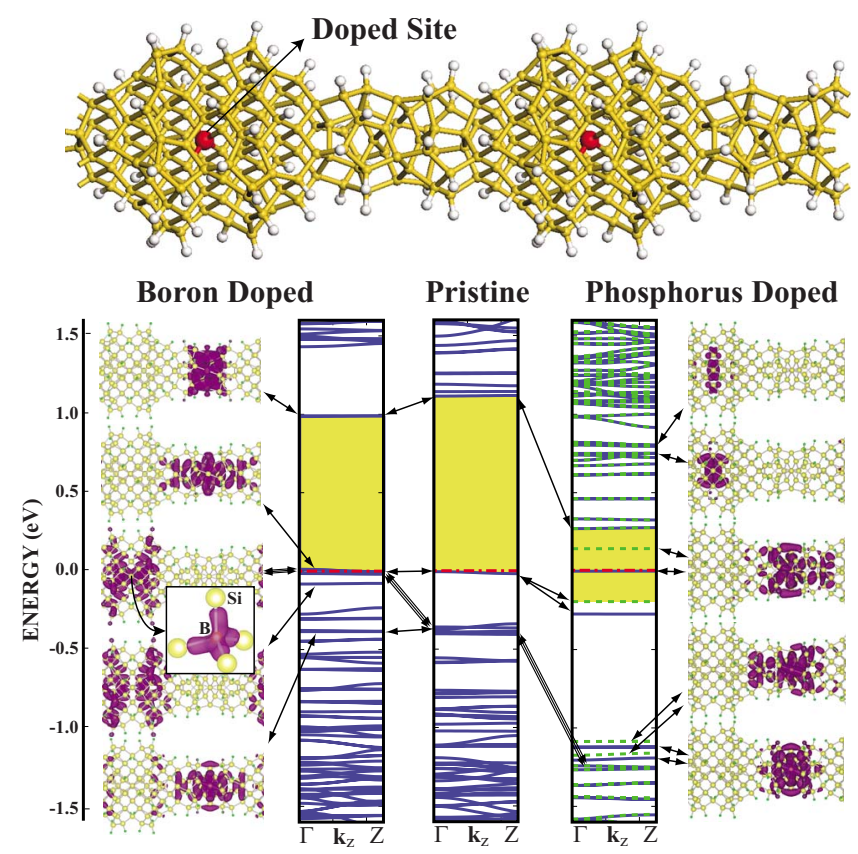

FIG. 5. (Color online) Atomic and electronic structure and charge-density isosurfaces of $\mathrm{Si}_{157} \mathrm{H}_{64}$ substitutional doped by $\mathrm{B}$ and $\mathrm{P}$ atoms [namely, $\mathrm{Si}_{156} \mathrm{P}(\mathrm{B}) \mathrm{H}_{64}$ ]. Top panel presents a view of the superlattice structure, where the doped site is represented by large red (dark) ball. Arrows between the bands of the undoped (pristine) superlattice and the bands of the B- and P-doped superlattices indicate the states, which preserved their character even after doping. The charge-density isosurfaces of specific states are linked to the corresponding bands by arrows. Fermi level, represented by dashed-dotted (red) line, was shifted to the valence-band edge and set to zero. Solid (blue) and dashed (green) lines represent the majority- and minority-spin states, respectively. Small panel at the left-hand side presents the charge-density isosurface plot of B-Si bonds.

It is known that substitutional B doping of bulk silicon crystal results in an acceptor state $\sim 45 \mathrm{meV}$ above the valence-band edge. ${ }^{22}$ In the substitutional doping of the superlattice by B similar situation occurs and the acceptor state is located above the valence-band edge corresponding to the wide part, but falls in the valence band of the narrow part. The filled first and the lower lying three valence bands at about $\sim-0.4 \mathrm{eV}$ undergo changes: the filled first valance band having states confined in the narrow part becomes half filled. Three lower lying valence bands of the pristine superlattice are raised toward the edge of the valence-band edge. The states of these bands mix with the tetrahedrally coordinated $s p^{3}$-hybridized orbitals of B and form a triply degenerate state just below the half-filled valence-band edge. Charge-density isosurfaces of these states around boron atom presented in Fig. 5 clearly indicates $s p^{3}$ hybridization in tetrahedral directions. As a result, the "holelike states" becomes confined in the narrow part. As for the conduction band is slightly shifted down but its states preserve their character.

In $\mathrm{P}$ doping of the superlattice at the center of the wide part, the impurity state localized at $\mathrm{P}$ atom occurs in the conduction band of the superlattice. This state, in fact, is the donor state occurring $\sim 200 \mathrm{meV}$ below the conduction 
band corresponding to the wide part. This energy is in good agreement with the energy calculated recently for the ionization energy of $\mathrm{P}$ donor states in the Si nanowires. ${ }^{23}$ Below this donor state, all conduction-band states are confined either in the narrow part or at the interface but not in the wide part. Energy shifts of the band-edge states are more pronounced in structure having substitutional $\mathrm{P}$ doping. Conduction-band edge is shifted down by $0.75 \mathrm{eV}$ but the charge-density profile remains the same. One spin-up and one spin-down bands split by $\sim 0.2 \mathrm{eV}$ are formed near the conduction-band edge with charge densities localized in the narrow part. The triply degenerate state in the valance band near the edge are shifted down. That states localized around substitutional P impurity appear in the conduction-band continua, while a filled spin-up band states are localized in the defect-free narrow part, indicate the possibility of modulation doping. In closing this section we note that the electronic structure associated with the doping of the small diameter superlattices is complex and has aspects different from that in the doping of bulk Si. The small size and dimensionality of the doped superlattices hinders the application of the effective-mass approximation leading to hydrogenic impurity model. For the same reason, the relative positions of band edges can be affected by the presence of a substitutional impurity.

\section{CONCLUSION}

In conclusion, we considered two specific superlattices grown in different crystallographic directions as a proof of concept. Each superlattice can be viewed to form the periodically repeating junctions of two hydrogen-saturated silicon nanowires of different diameters. Since the band gaps of the constituent Si nanowires are different, the band gaps of these superlattices are modulated in real space. As a result, one-dimensional multiple quantum-well structures with states confined in different (wide or narrow) parts are formed. At strong confinement or localization, the superlattice structures can be viewed as a series of quantum dots. We believe that the band-gap modulation and resulting quantumwell structure and confinement can occur as long as the band-gap differences are maintained between adjacent region at both sides of the junction. Furthermore, we showed that the electronic structure resulting from the substitutional doping of the superlattice is interesting and gives rise to modulation doping. We finally note that, the band gaps of SiNWs at both sides of junction forming the superlattice structure are underestimated by DFT/generalized gradient approximation (GGA) calculations. Since the systems under study are too large, these bands cannot be improved by GW calculations. Nevertheless, our work is just a proof of concept. According to our results, if the band gaps are different in different parts of the heterostructure, confined states can occur and modulation doping of these quantum structures can be achieved. Details of energy-band structure and location of impurity states in the gap should only be taken qualitatively.

Various types of electronic devices, such as resonant tunneling double barriers generated from these superlattices, can be arranged on a single rod, where they can be connected by the metallic segments of unsaturated Si nanowires. The character of these devices can be engineered by varying their growth direction and structural parameters, such as $l_{1}, l_{2}, D_{1}$, and $D_{2}$. Even if the diameters of superlattices we treated in this study are small $(\sim 1 \mathrm{~nm})$ and hence in the range where band gaps vary significantly with diameter, they are still in the range of those fabricated recently. ${ }^{1}$

\section{ACKNOWLEDGMENTS}

Part of the computations have been carried out by using UYBHM at Istanbul Technical University through a Grant No. 2-024-2007. This work is partially supported by TÜBA, Academy of Science of Turkey.

\footnotetext{
*ciraci@fen.bilkent.edu.tr

${ }^{1}$ D. D. D. Ma, C. S. Lee, F. C. K. Au, S. Y. Tong, and S. T. Lee, Science 299, 1874 (2003).

${ }^{2}$ A. K. Singh, V. Kumar, R. Note, and Y. Kawazoe, Nano Lett. 6, 920 (2006).

${ }^{3}$ Y. Cui, Z. Zhong, D. Wang, W. U. Wang, and C. M. Lieber, Nano Lett. 3, 149 (2003).

${ }^{4}$ Y. Huang, X. F. Duan, and C. M. Lieber, Small 1, 142 (2005).

${ }^{5}$ X. F. Duan, Y. Huang, R. Agarwal, and C. M. Lieber, Nature (London) 421, 241 (2003).

${ }^{6}$ Y. Cui, X. F. Duan, J. Hu, and C. M. Lieber, J. Phys. Chem. B 104, 5213 (2000).

${ }^{7}$ M. V. Fernandez-Serra, Ch. Adessi, and X. Blase, Phys. Rev. Lett. 96, 166805 (2006).

${ }^{8}$ E. Durgun, D. Cakir, N. Akman, and S. Ciraci, Phys. Rev. Lett. 99, 256806 (2007); E. Durgun, N. Akman, and S. Ciraci, Phys. Rev. B 78, 195116 (2008).

${ }^{9}$ M. S. Gudiksen, L. J. Lauhon, J. Wang, D. C. Smith, and C. M.
}

Lieber, Nature (London) 415, 617 (2002)

${ }^{10}$ M. T. Bjork, B. J. Ohlsson, T. Sass, A. I. Persson, C. Thelander, M. H. Magnusson, K. Deppert, L. R. Wallenberg, and L. Samuelson, Nano Lett. 2, 87 (2002).

${ }^{11}$ Y. Wu, R. Fan, and P. Yang, Nano Lett. 2, 83 (2002).

${ }^{12}$ N. Akman, E. Durgun, S. Cahangirov, and S. Ciraci, Phys. Rev. B 76, 245427 (2007).

${ }^{13}$ W. Kohn and L. J. Sham, Phys. Rev. 140, A1133 (1965); P. Hohenberg and W. Kohn, Phys. Rev. 136, B864 (1964).

${ }^{14}$ D. Vanderbilt, Phys. Rev. B 41, 7892 (1990).

${ }^{15}$ G. Kresse and J. Hafner, Phys. Rev. B 47, 558 (1993); G. Kresse and J. Furthmuller, ibid. 54, 11169 (1996).

${ }^{16}$ J. P. Perdew, J. A. Chevary, S. H. Vosko, K. A. Jackson, M. R. Pederson, D. J. Singh, and C. Fiolhais, Phys. Rev. B 46, 6671 (1992).

${ }^{17}$ L. Esaki and L. L. Chang, Phys. Rev. Lett. 33, 495 (1974); Highlights in Condensed Matter Physics and Future Prospects NATO Science Forum Series B Vol. 285, edited by L. Esaki, 
(Plenum, New York, 1991).

${ }^{18}$ X. Zhao, C. M. Wei, L. Yang, and M. Y. Chou, Phys. Rev. Lett. 92, 236805 (2004); M. Bruno, M. Palummo, A. Marini, R. DelSole, and S. Ossicini, ibid. 98, 036807 (2007); R. Rurali, B. Aradi, Th. Frauenheim, and A. Gali, Phys. Rev. B 76, 113303 (2007).

${ }^{19}$ I. Ponomareva, E. Richter, A. N. Andriotis, and Madhu Menon, Nano Lett. 7, 3424 (2007).

${ }^{20}$ S. Ciraci and I. P. Batra, Phys. Rev. B 36, 6194 (1987); E. Tekman, S. Ciraci, and A. Baratoff, ibid. 42, 9221 (1990).
${ }^{21}$ M. P. Persson, A. Lherbier, Y. Niquet, F. Triozon, and S. Roche, Nano Lett. 8, 4146 (2008).

${ }^{22}$ Handbook of Semiconductor Silicon Technology, edited by W. C. O'Mara, R. B. Herring, and L. P. Hunt (Noyes, New Jersey, 1990).

${ }^{23}$ M. Diarra, Y.-M. Niquet, C. Delerue, and G. Allan, Phys. Rev. B 75, 045301 (2007); T.-L. Chan, M. L. Tiago, E. Kaxiras, and J. R. Chelikowsky, Nano Lett. 8, 596 (2008); C. R. Leao, A. Fazzio, and A. J. R. daSilva, Nano Lett. 8, 1866 (2008). 